\title{
THE AUTOMORPHISM GROUP OF A GEOMETRIC STRUCTURE \\ BY
}

\author{
HSIN CHU AND SHOSHICHI KOBAYASHI(1)
}

Given a geometric structure on a manifold $M$, the group of transformations of $M$ leaving the structure invariant is often a Lie transformation group $\left({ }^{2}\right)$. In this report we shall give a historical account of such cases and systematic proofs of those results. In $\$ 1$ we summarize known results in the chronological order and in $\$ 2$ we show how to derive them from a theorem of Palais. We give also a self-contained proof of the result of Palais as it is not easy to pick up the proof from his long paper.

1. A summary of known results. In 1935, H. Cartan [8] proved:

THEOREM A. The group $G$ of holomorphic transformations, with compactopen topology, of a bounded domain $M$ in $C^{n}$ is a Lie transformation group. Moreover, the isotropy subgroup at each point of $M$ is compact.

His proof may be described as follows. Let $\phi_{1}, \phi_{2}, \cdots\left(\phi_{k} \neq\right.$ identity transformation) be a sequence of holomorphic transformations of $M$ converging to the identity transformation with respect to the compact-open topology. Then there exist a subsequence $\left\{\phi_{k_{l}}\right\}$ and a sequence of positive integers $\left\{m_{i}\right\}$ such that the sequence

$$
m_{i}\left(\phi_{k_{i}}(z)-z\right), \quad z=\left(z^{1}, \cdots, z^{n}\right) \in C^{n}
$$

converges to a nonzero holomorphic function $\zeta=\left(\zeta^{1}, \cdots, \zeta^{n}\right)$. The holomorphic vector field $\Sigma \zeta^{i}\left(\partial / \partial z^{i}\right)$ generates a global 1-parameter group of holomorphic transformations of $M$. On the other hand, choose a linear frame $u_{0}$ of $M$ once and for all. For each holomorphic transformation $\phi$ of $M$, let $\phi\left(u_{0}\right)$ be the image frame. Then $\phi \rightarrow \phi\left(u_{0}\right)$ is a one-to-one mapping of $G$ into the bundle of linear frames of $M$ and its image is closed. From these two facts, Cartan derives Theorem A.

In 1939, Myers and Steenrod [19] obtained:

Received by the editors November 1, 1962 and, in revised form, May 22, 1963.

(1) Supported by NSF contract GP-812.

(2) For certain $G$-structures of infinite type, e.g., symplectic structures and contact structures, the automorphism groups are always too large to be Lie groups; P. Libermann, Sur les automorphismes infinitésimaux des structures symplectiques et des structures de contact, Colloque de Géométrie Différentielle Globale, Brussels 1958, pp. 37-59. 
THEOREM B. The group $I(M)$ of isometries of a Riemannian manifold $M$ is a Lie transformation group with respect to the compact-open topology.

Let $n=\operatorname{dim} M$. They proved that, for suitably chosen $n+1$ points $x_{0}, x_{1}, \cdots, x_{n} \in M$, the mapping $I(M) \rightarrow M^{n+1}=M \times \cdots \times M(n+1$ times $)$ which sends $\phi \in I(M)$ into $\left(\phi\left(x_{0}\right), \phi\left(x_{1}\right), \cdots, \phi\left(x_{n}\right)\right)$ is one-to-one and that its image is a closed submanifold of $M^{n+1}$. Then they show that, with respect to the differentiable structure thus introduced, $I(M)$ is a Lie group acting differentiably on $M$.

We should perhaps mention at this point the following result due to van Dantzig and van der Waerden [10]:

The group of isometries of a connected, locally compact metric space is locally compact with respect to the compact-open topology.

In 1946, Bochner and Montgomery [5] obtained:

THEOREM C. Let $G$ be a locally compact group of differentiable transformations of class $C^{2}$ acting effectively on a differentiable manifold $M$ of class $C^{2}$. Then $G$ is a Lie transformation group.

Making use of Bochner's result [1] on compact groups of differentiable transformations, they proved the nonexistence of small subgroups. Following Cartan's argument, they constructed 1-parameter subgroups. The finite dimensionality of $G$ (which, in Theorems $\mathrm{A}$ and $\mathrm{B}$, was a consequence of the fact that $G$ is imbeddable in a certain manifold) was proved by means of a formula obtained in their earlier paper [4].

REMARK. In [5] they assumed that an element of $G$ which fixes a nonempty open subset of $M$ is the identity element. This assumption can be removed by using Bochner's result [1] (cf. the book of Montgomery-Zippin [18, p. 208]). In 1950, Kuranishi [17] proved the above theorem of Bochner-Montgomery under the assumption of difierentiability of class $C^{1}$.

As a corollary to Theorem $\mathrm{C}$, Bochner and Montgomery obtained:

THEOREM D. The group $G$ of holomorphic transformations of a compact complex manifold $M$ is a Lie transformation group.

From properties of holomorphic functions, $G$ is easily seen to be locally compact with respect to the compact-open topology and Theorem $C$ applies.

Later they proved [6]:

THEOREM E. The group $G$ of holomorphic transformations of a compact complex manifold $M$ is a complex Lie group and the action $G \times M \rightarrow M$ is holomorphic.

Generalizing the result of Myers and Steenrod, in 1953 Nomizu [21] proved:

THEOREM F. Let $M$ be a manifold with an affine connection. Then the group $A(M)$ of affine transformations of $M$ is a Lie transformation group. 
Working on the base manifold $M$ and its tangent bundle, he proved that $A(M)$ is locally compact with respect to the compact-open topology, thus reducing the problem to Theorem $C$. Originally, he had to assume that the connection is complete. Later he was able to remove the assumption by considering every affine transformation $\phi$ of $M$ as an isometry on the bundle $L(M)$ of linear frames. (If $\theta=\left(\theta^{i}\right)$ is the canonical form on $L(M)$ and $\omega=\left(\omega_{k}^{j}\right)$ is the connection form on $L(M)$, then the natural prolongation of $\phi$ to $L(M)$ leaves the Riemannian metric $d s^{2}=\Sigma_{i}\left(\theta^{i}\right)^{2}+\Sigma_{j, k}\left(\omega_{k}^{j}\right)^{2}$ invariant.) Thus he reduced the proof of Theorem F to Theorem B. Independently, Hano and Moritomo [13] were also successful in removing the assumption of completeness; they proved that $G$ is locally compact, also making use of the Riemannian metric on $L(M)$ constructed above.

In 1954, Kobayashi [14] (see also [15]) proved the following theorem and derived, as immediate consequence, the result of Myers-Steenrod and that of Nomizu.

THEOREM G. Let $M$ be an n-dimensional manifold with $n$ 1-forms $\alpha_{1}, \cdots, \alpha_{n}$ which are linearly independent at each point of $M$. Then the group $G$ of transformations of $M$ leaving $\alpha_{1}, \cdots, \alpha_{n}$ invariant is a Lie transformation group.

The proof is similar to, but simpler than, that of Myers-Steenrod's theorem as the assumption is stronger. He proved that, if $x_{0}$ is an arbitrary point of $M$, then $\phi \rightarrow \phi\left(x_{0}\right)$ is a one-to-one mapping of $G$ into $M$ and its image is a closed submanifold of $M$ and that, with respect to the differentiable structure thus introduced in $G$, the action is differentiable.

Although Theorem $G$ seems to be a special case of Theorem B, it actually implies Theorems $\mathrm{B}$ and $\mathrm{F}$. Let $M$ be a manifold with an affine connection. Let $\theta=\left(\theta^{i}\right)$ and $\omega=\left(\omega_{k}^{j}\right)$ be the canonical form and the connection form on $L(M)$ as before. Then $n+n^{2} 1$-forms $\theta^{i}, \omega_{k}^{j}$ are linearly independent on the $\left(n+n^{2}\right)$-dimensional manifold $L(M)$ and the natural prolongation to $L(M)$ of every affine transformation $\phi$ of $M$ leaves these 1-forms invariant. Conversely, every fibre preserving transformation of $L(M)$ leaving these 1-forms invariant is the natural prolongation of an affine transformation $\phi$ of $M$. Thus, the group $A(M)$ of affine transformations of $M$ can be considered as a closed subgroup of the group of transformations of $L(M)$ leaving these 1 -forms invariant. Since the latter is a Lie transformation group by Theorem $\mathrm{G}$, so is $A(M)$. Theorem B can be derived similarly by applying Theorem $\mathrm{G}$ to the bundle $O(M)$ of orthonormal frames of a Riemannian manifold $M$ and to the $\frac{1}{2} n(n+1) 1$-forms $\theta^{i}, \omega_{k}^{j}$, where $1 \leqq i \leqq n$ and $1 \leqq j<k \leqq n$. Or, Theorem $\mathrm{B}$ can be immediatey derived from Theorem F.

In 1957, Palais obtained the following general result [23, p. 103]:

THEOREM H. Let $G$ be a group of diffeomorphisms of a manifold $M$. Let $S$ be the set of all vector fields $X$ on $M$ which generate global 1-parameter groups 
$\phi_{t}=\exp t X$ of transformations of $M$ such that $\phi_{t} \in G$. If $S$ generates a finitedimensional Lie algebra, then $G$ is a Lie transformation group and $S$ is the Lie algebra of $G$.

The proof of this theorem will be reproduced in $\$ 2$.

Generalizing Theorem A, Kobayashi [16] proved:

THEOREM I. If $M$ is an $n$-dimensional complex manifold with sufficiently many square integrable holomorphic n-formas, then the group $G$ of holomorphic transformations of $M$ is a Lie transformation group and the isotropy subgroup of $G$ at every point of $M$ is compact.

A holomorphic $n$-form $f$ on $M$ is square integrable if

$$
\int_{M}(\sqrt{ }(-1))^{n^{2}} f \wedge \bar{f}<\infty
$$

Let $F$ be the Hilbert space consisting of all such $n$-forms $f$. By "sufficiently many", we mean that

(1) At each point $x \in M$, there exists an $f \in F$ such that $f(z) \neq 0$.

(2) If $z^{1}, \cdots, z^{n}$ is a local coordinate system in a neighborhood of a point $x \in M$, then, for each $j$, there exists an

$$
h=h^{*} d z^{1} \wedge \cdots \wedge d z^{n} \in F
$$

such that $h^{*}(x)=0$ and $\left(\partial h^{*} / \partial z^{j}\right)_{x} \neq 0$.

Let $h_{0}, h_{1}, h_{2}, \cdots$ be an orthonormal basis for $F$ and set

$$
K=K^{*} d z^{1} \wedge \cdots \wedge d z^{n} \wedge d \bar{z}^{1} \wedge \cdots \wedge d \bar{z}^{n}=\sum_{i=0}^{\infty} h_{i} \wedge h_{i} .
$$

Then the Bergman metric given by

$$
d s^{2}=\sum_{\alpha, \beta=1}^{n} g_{\alpha \beta} d z^{\alpha} d \bar{z}^{\beta}, \quad \text { where } g_{\alpha \bar{\beta}}=\partial^{2} \log K^{*} / \partial z^{\alpha} \partial \bar{z}^{\beta},
$$

is invariant by $G$. It follows that $G$ is a closed subgroup of the group $I(M)$ of isometries of $M$ and hence is a Lie transformation group by Theorem B.

Recently, Boothby-Kobayashi-Wang [7] obtained the following generalization of Theorem D.

THEOREM J. The automorphism group $G$ of a compact almost complex manifold $M$ is a Lie transformation group.

They found a system of elliptic partial differential equations satisfied by almost complex mappings. The result of Douglis-Nirenberg [11] implies that $G$ is locally compact and hence is a Lie transformation group by Theorem $C$.

2. Proofs of Theorems A, B, D, E, F, G, H, I and J. We shall proceed in the following order: 
(1) $\mathrm{H} \rightarrow \mathrm{G} \rightarrow \mathrm{F} \rightarrow \mathrm{B} \rightarrow \mathrm{I} \rightarrow \mathrm{A}$;

(2) $\mathrm{H} \rightarrow \mathrm{J} \rightarrow \mathrm{D} \rightarrow \mathrm{E}$.

What we actually prove here are Theorem $\mathrm{H}$ and the implications $\mathrm{H} \rightarrow \mathrm{G}, \mathrm{H} \rightarrow \mathrm{J}$ and $\mathrm{D} \rightarrow \mathrm{E}$. The other implications are either evident or have been explained in $\$ 1$. In proving the implication $\mathbf{H} \rightarrow \mathrm{J}$ we make use of Bochner's theorem on the finite dimensionality of the space of tensor fields satisfying a system of elliptic partial differential equations on a compact manifold. Otherwise, our proofs are self-contained.

Proof of Theorem H. Let $\mathrm{g}^{*}$ be the Lie algebra generated by $S$ and let $\tilde{G}$ be the connected, simply connected Lie group generated by $\mathrm{g}^{*}$. For each $X \in \mathrm{g}^{*}$, denote by $\exp t X$ the 1-parameter subgroup of $\widetilde{G}$ generated by $X$.

Lemma 1. If $X, Y \in S$, then $(\operatorname{ad}(\exp X)) Y \in S$.

Proof of Lemma 1. Set $Z=(\operatorname{ad}(\exp \mathrm{X})) Y$. Then

$$
\exp t Z=(\exp X)(\exp t Y)(\exp X)^{-1},
$$

which shows that $(\exp t Z) x$ is defined for all $x \in M$ and all $t,-\infty<t<\infty$.

Lemma 2. $S$ spans $\mathrm{g}^{*}$.

Proof of Lemma 2. Let $V$ be the linear subspace of $\mathrm{g}^{*}$ spanned by $S$. By Lemma 1, we have $(\operatorname{ad}(\exp S)) S \subset S$ and, hence, $(\operatorname{ad}(\exp S)) V \subset V$. Since $S$ generates $\mathfrak{g}^{*}$, $\exp S$ generates $\tilde{G}$. Hence, $(\operatorname{ad} \tilde{G}) V \subset V$. In particular, $(\operatorname{ad}(\exp V)) V \subset V$. This implies that $[V, V] \subset V$, i.e., $V$ is a subalgebra of $\mathrm{g}^{*}$. Since $V$ contains $S, V$ generates $\mathrm{g}^{*}$ It follows that $V=\mathrm{g}^{*}$.

Lemma 3. $S=\mathrm{g}^{*}$.

Proof of Lemma 3. Let $X_{1}, \cdots, X_{r} \in S$ be a basis for $g^{*}$. Then the mapping

$$
\mathrm{g}^{*} \ni \Sigma a^{i} X_{i} \rightarrow\left(\exp a^{1} X_{1}\right) \cdots\left(\exp a^{r} X_{r}\right) \in \tilde{G}
$$

gives a diffeomorphism of a neighborhood $N$ of 0 in $\mathrm{g}^{*}$ onto a neighborhood $U$ of the identity in $\hat{G}$. Let $Y \in \mathrm{g}^{*}$. Let $\delta$ be a positive number such that $\exp t Y \in U$ for $|t|<\delta$. Then, for each $t$ with $|t|<\delta$, there exists a unique $\Sigma a^{i}(t) X_{i} \in N$ such that

$$
\exp t Y=\left(\exp a^{1}(t) X_{1}\right) \cdots\left(\exp a^{r}(t) X_{r}\right) .
$$

The action of $\exp t Y$ on $M$ is therefore given by

$$
(\exp t Y) x=\left(\exp a^{1}(t) X_{1}\right) \cdots\left(\exp a^{r}(t) X_{r}\right) x \quad \text { for } x \in M \text { and }|t|<\delta .
$$

This shows that every element $Y$ of $g^{*}$ generates a global 1-parameter group of transformations of $M$, thus completing the proof of Lemma 3 . 
Let $G^{*}$ be the Lie transformation group acting on $M$ generated by $\mathfrak{g}^{*}$. Since every element of $\mathrm{g}^{*}$ generates a global 1-parameter group of transformations of $M$ by Lemma 3, $G^{*}$ exists. Since $G^{*}$ is connected, the assumption in Theorem $\mathrm{H}$ implies that $G^{*} \subset G$. Let $\phi \in G$ and let $\psi_{t}$ be a 1-parameter subgroup of $G^{*}$. Then $\phi \cdot \psi_{t} \cdot \phi^{-1}$ is a 1-parameter group of transformations of $M$ contained in $G$. From the construction of $G^{*}$ it follows that this 1-parameter group is a subgroup of $G^{*}$. Since $G^{*}$ is generated by its 1-parameter subgroups, this implies that $G^{*}$ is a normal subgroup of $G$. Each $\phi \in G$ defines an automorpbism $A_{\phi}: G^{*} \rightarrow G^{*}$ by $A_{\phi}(\psi)=\phi \cdot \psi_{t} \cdot \phi^{-1}$. Since $A_{\phi}$ sends every 1-parameter subgroup of $G^{*}$ into a 1-parameter subgroup of $G^{*}$, it is continuous (cf. Chevalley's book $[9$, p. 128]).

LEMMA. Let $G$ be a group and $G^{*}$ a topological group which is contained in $G$ as a normal subgroup. If $A_{\phi}: G^{*} \rightarrow G^{*}$ is continuous for each $\phi \in G$, then there exists a unique topology on $G$ which makes $G^{*}$ open in $G$.

Proof of lemma. If $\{V\}$ is the system of open neighborhoods of the identity in $G^{*}$, we take $\{\phi(V)\}$ as the system of open neighborhoods of $\phi \in G$ in $G$. It is a trivial matter to verify that $G^{*}$ is open in $G$ with respect to the topology thus defined in $G$. The uniqueness of such a topology is also evident.

This lemma applied to our case, we have a topological group $G$ and a normal subgroup $G^{*}$ which is a connected Lie group. Thus $G^{*}$ is the connected component of the identity of $G$. In an obvious manner, we introduce a differentiable structure in $G$. It is a routine matter to verify that $G$ is a Lie group and that the differentiability of the action $G^{*} \times M \rightarrow M$ implies the differentiability of the action $G \times M \rightarrow M$. This completes the proof of Theorem $\mathrm{H}$.

Proof of Theorem G. Let $X_{1}, \cdots, X_{n}$ be the vector fields on $M$ defined by $\alpha^{i}\left(X_{j}\right)=\delta_{j}^{i}$, so that $X_{1}, \cdots, X_{n}$ are linearly independent at each point of $M$. A transformation $\phi$ of $M$ leaves $\alpha_{1}, \cdots, \alpha_{n}$ invariant if and only if it leaves $X_{1}, \cdots, X_{n}$ invariant. Let $Y$ be a vector field on $M$ which generates a 1-parameter group of global transformations of $M$. This 1-parameter group leaves $X_{1}, \cdots, X_{n}$ invariant if and only if $\left[Y, X_{j}\right]=0$ for $j=1, \cdots, n$. Let $\mathfrak{a}$ be the set of all vector fields $Y$ on $M$ such that $\left[Y, X_{j}\right]=0$ for $j=1, \cdots, n$. We see that $\mathfrak{a}$ is a Lie algebra. In view of Theorem $\mathrm{H}$, it is sufficient to show that $\operatorname{dim} \mathfrak{a}$ is finite. Let $Y$ be any nonzero element of $\mathfrak{a}$. We shall show that the vector field $Y$ vanishes nowhere on $M$. Let $Z_{Y}$ be the set of zeros of $Y$, i.e., $Z_{Y}=\left\{x \in M ; Y_{x}=0\right\}$. Evidently, $Z_{Y}$ is closed in $M$. For each set of $n$ numbers $u^{1}, \cdots, u^{n}$, we set $X=\sum_{i=1}^{n} u^{i} X_{i}$ and let $\phi_{t}=\exp t X$ be the local transformations of $M$ generated by $X$. For each point $x \in Z_{Y},\left\{\phi_{t}(x) ;\left|u^{i}\right|<\delta,|t|<1\right\}$ covers a neighborhood $U$ of $x$, where $\delta$ is a small positive number. Since $\left[Y, \Sigma u^{i} X_{i}\right]=0, \phi_{t}$ leaves $Y$ invariant. Hence $\phi_{t}\left(Y_{x}\right)=Y_{\phi_{t}}(x)=0$. This means that $Y$ vanishes at every point of $U$. Thus, $Z_{Y}$ is open in $M$. The connectedness of $M$ implies that $Y$ is nonzero at every point of $M$. Therefore, for any point $x$ of $M$, the mapping $a \rightarrow T_{x}(M)$ (the tangent space 
of $M$ at $x$ ) which sends $Y$ into $Y_{x}$ is one-to-one. In particular, we have $\operatorname{dim} \mathfrak{a} \leqq \operatorname{dim} M=n$. This completes the proof of Theorem $\mathrm{G}$.

Proof of Theorem J. We first state a special case of a theorem of Bochner [3].

THEOREM OF BOCHNER. Let $S$ be a space of vector fields $X$ on a compact manifold $M$ such that, for every point of $M$, there is a system of elliptic partial differential equations

$$
\sum_{j, k} g^{j k} \frac{\partial^{2} \xi^{i}}{\partial x^{j} \partial x^{k}}+\sum_{j, k} h_{j}^{i k} \frac{\partial \xi^{j}}{\partial x^{k}}+\sum_{j} h_{j}^{i} \xi^{j}=0, \quad i=1, \cdots, n,
$$

defined in a neighborhood of that point and satisfied by all

$$
X=\sum_{i} \xi^{i} \frac{\partial}{\partial x^{i}}
$$

Then the dimension of $S$ is finite.

We first recall the definition of the torsion tensor $T$ and the curvature tensor $R$ of an affine connection in terms of covariant differentiation $\nabla$. For any vector fields $X, Y$ and $Z$ on $M$, we have [22]:

(1) $T(X, Y)=\nabla_{X} Y-\nabla_{Y} X-[X, Y]$,

(2) $R(X, Y)=\left[\nabla_{X}, \nabla_{Y}\right] Z-\nabla_{[X Y]} Z$.

Let $M$ be a manifold with almost complex structure $J ; J$ is a tensor field of type $(1,1)$ (i.e., contravariant degree 1 and covariant degree 1) or a field of endomorphisms such that $J^{2}=-1$. A vector field $X$ is an infinitesimal automorphism of the almost complex structure $J$ if and only if

(3) $[X, J V]=J([X, V])$ for all vector fields $V$.

Consider a hermitian metric $g$ on $M$; it is a Riemannian metric such that $g\left(J V, J V^{\prime}\right)=g\left(V, V^{\prime}\right)$ for all vector fields $V$ and $V^{\prime}$. Consider an affine connection on $M$ such that $\nabla g=0$ and $\nabla J=0$; such a connection can always be obtained by considering a connection in the bundle of unitary frames over $M$.

We shall now construct a system of elliptic partial differential equations satisfied by all infinitesimal automorphisms $X$ of $J$. From (1) we obtain

(4) $[X, J V]=\nabla_{X}(J V)-\nabla_{J V} X-T(X, J V)$,

(5) $J([X, V])=J\left(\nabla_{X} V\right)-J\left(\nabla_{V} X\right)-J(T(X, V))$,

where $X$ and $V$ are arbitrary. Since $\nabla J=0, \nabla_{X}(J V)=J\left(\nabla_{X} V\right)$. By (3), (4) and (5), we see that $X$ is an infinitesimal automorphism of $J$ if and only if we have

(6) $\nabla_{J V} X-J\left(\nabla_{V} X\right)+B(V, X)$ for all vector fields $V$, where $B$ is the tensor field of type $(1,2)$ defined by

$$
B(V, X)=T(X, J V)-J(T(X, V)) .
$$

Applying $J$ to (6), we see that (6) is equivalent to the following: 
(7) $\nabla_{V} X+J\left(\nabla_{J V} X\right)+J(B(V, X))=0$ for all vector fields $V$.

Apply $\nabla_{V}$ to (6) and $\nabla_{J V}$ to (7). We then see that if $X$ is an infinitesimal automorphism of $J$, then

(8) $\nabla_{V} \cdot \nabla_{V} X+J\left(\nabla_{V} \cdot \nabla_{J V} X\right)+\nabla_{V}(J(B(V, X)))=0$ for all vector fields $V$,

(9) $\nabla_{J V} \cdot \nabla_{J V} X-J\left(\nabla_{J V} \cdot \nabla_{V} X\right)+\nabla_{J V}(B(V, X))=0$ for all vector fields $V$. Since we have (cf. (2)):

$$
\left(\nabla_{V} \nabla_{J V}-\nabla_{J V} \nabla_{V}\right) X=R(V, J V) X+\nabla_{[V, J V]} X,
$$

by adding (8) and (9) we obtain:

(10) $\left(\nabla_{V} \cdot \nabla_{V}+\nabla_{J V} \cdot \nabla_{J V}\right) X+\cdots=0$ for all vector fields $V$, where the dots $\cdots$ indicate terms not involving second derivatives of $X$. Locally, we choose vector fields $V_{1}, \cdots, V_{n}$ such that $V_{1}, \cdots, V_{n}, J V_{1}, \cdots, J V_{n}$ are orthonormal. Apply (10) to each $V_{i}$ and then sum over $i=1, \cdots, n$. Then we have a system of elliptic partial differential equations satisfied by all infinitesimal automorphisms $X$ of $J$.

By Bochner's theorem, if $M$ is compact, the Lie algebra of infinitesimal automorphisms $X$ of $J$ is finite-dimensional. By Theorem $\mathrm{H}$, the group of automorphisms of a compact complex manifold $M$ is a Lie transformation group.

Proof of Theorem E. Let $M$ be a compact complex manifold. Then the Lie algebra of holomorphic vector fields is precisely the Lie algebra of infinitesimal automorphisms of the complex structure $J$. Since $M$ is compact, every vector field on $M$ generates a 1-parameter group of global transformations of $M$. Let $G^{*}$ be the Lie transformation group of $M$ generated by the Lie algebra of all holomorphic vector fields of $M$. Then $G^{*}$ is obviously a complex Lie group and the group action $G^{*} \times M \rightarrow M$ is holomorphic. From the proof of Theorem $\mathbf{H}$, it follows that the group $G$ of holomorphic transformations of $M$ is a complex Lie group whose identity component is $G^{*}$ and that the group action $G \times M \rightarrow M$ s also holomorphic.

3. Concluding remarks. 1. In Theorem $G$, the dimension of the group $G$ is at most $n=\operatorname{dim} M$, and when this maximum is attained, $G$ can be identified with $M$. In Theorem $\mathrm{F}$, the dimension of the group $A(M)$ is at most $n+n^{2}$, and when this maximum is attained, $M$ is an affine space. Similarly, in Theorem $\mathbf{B}$, the dimension of the group $I(M)$ is not greater than $\frac{1}{2} n(n+1)$, and when this maximum is attained $M$ is either a simply connected complete space of constant curvature or a real projective space (with constant positive curvature). In Theorem A or Theorem I, the group $G$ attains the maximum dimension $n(n+2)$ only when $M$ is a simply connected, complete Kaehler manifold of constant negative holomorphic curvature. On the other hand, in Theorem $\mathrm{D}$ and Theorem $\mathrm{J}$, the maximum dimension of $G$ is not known. It is reasonable to conjecture that the maximum is $2 n(n+2)$ and is attained only when $M$ is a complex projective space.

2. In the case of a bounded domain in $C^{n}$, if $X$ is an infinitesimal automorphism which generates a 1-parameter group of global transformations of $M$, then $J X$ does 
not generate a 1-parameter group of global transformations of $M$. This explains why no complex Lie group acts holomorphically on a bounded domain of $C_{n}$ (see $[8 ; 16])$.

3. Given an almost complex structure $J$, let $N$ be the tensor field of type $(1,2)$ defined by

$$
N(X, Y)=[X, Y]+J([J X, Y])+J([X, J Y])-[J X, J Y]
$$

where $X$ and $Y$ are arbitrary vector fields on $M$. By a theorem of NewlanderNirenberg [20], $N=0$ if and only if $J$ comes from a complex structure. From (3) in the proof of Theorem $\mathrm{J}$, it follows that, given a vector field $X$ on $M$, both $X$ and $J X$ are infinitesimal automorphisms of the almost complex structure $J$ if and only if $N(X, V)=0$ for all vector fields $V$ on $M$.

It follows that if $G$ is a complex Lie group acting transitively on an almost complex manifold $M$ in such a way that the action $G \times M \rightarrow M$ is almost complex, then $M$ is actually a complex manifold. This shows that Theorem E cannot be generalized to almost complex manifolds.

4. The system of elliptic partial differential equations obtained in the proof of Theorem $\mathrm{J}$ can be explicitly expressed in terms of the metric tensor, the tensor $J$, the torsion $T$ and the curvature $R$ (of the affine connection we used). When $M$ is a Kaehler manifold, it reduces to the following equation of Bochner [2]:

$$
\sum_{j, k} g^{j k} \xi_{; j ; k}^{i}+\sum_{j} R_{j}^{i} \xi^{j}=0
$$

Yano [25] proved that, for a compact Kaehler manifold, every solution of the above equation of Bochner is necessarily an infinitesimal holomorphic transformation. It seems difficult to generalize Yano's result to our case.

5. In this report, by "an isometry of a Riemannian manifold" we meant a diffeomorphism of the manifold which preserves the metric tensor. The distance between two points $x$ and $y$ of a Riemannian manifold $M$ is defined to be the infimum of the arc-lengths of all piecewise differentiable curves joining $x$ and $y$. It is known [19] (see also [24]) that a mapping of $M$ into itself which preserves distance is necessarily a diffeomorphism and preserves the metric tensor.

6. Theorem F can be generalized to conformal connections, projective connections and, more generally, to any Cartan connection in the sense of Ehresmann since each Cartan connection defines an absolute parallelism in the principal fibre bundle considered [12], [15].

\section{BIBLIOGRA PHY}

1 S. Bochner, Compact groups of differentiable transformations, Ann. of Math. (2) 46 (1945), 372-381.

2. - Vector fields and Ricci curvature, Bull. Amer. Math. Soc. 52 (1946), 776-797.

3. —_, Tensor fields with finite bases, Ann. of Math. (2) 53 (1951), 400-411. 
4. S. Bochner and D. Montgomery, Groups of differentiable and real or complex analytic transformations, Ann. of Math. (2) 46 (1945), 685-694.

5. - Locally compact groups of differentiable transformations, Ann. of Math. (2) 47 (1946), 639-653.

6. - Groups on analytic manifolds, Ann. of Math. (2) 48 (1947), 659-669.

7. W. Boothby, S. Kobayashi and H. C. Wang, A note on mappings and automorphisms of almost complex manifolds, Ann. of Math. (2) 77 (1963), 329-334.

8. H. Cartan, Sur les groups de transformations analytiques, Actualités Sci. Ind. No. 198, Hermann, Paris, 1935.

9. C. Chevalley, Theory of Lie groups, Princeton Univ. Press, Princeton, N. J., 1946.

10. D. van Dantzig and B. L. van der Waerden, Über metrisch homogene Räume, Abh. Math. Sem. Univ. Hamburg 6 (1928), 374-376.

11. A. Douglis and L. Nirenberg, Interior estimates for elliptic system of partial differential equations, Comm. Pure Appl. Math. 8 (1955), 503-538.

12. C. Ehresmann, Les connexions infinitésimales dans un espace fibré différentiable, Colloque de Topologie (Brussels, 1950), Thone, Liège, 1951; pp. 29-55.

13. J. Hano and A. Morimoto, Note on the group of affine transformations of an affinely connected manifold, Nagoya Math. J. 8 (1955), 71-81.

14. S. Kobayashi, Le groupe de transformations qui laissent invariant un parallélisme, Colloque de Topologie (Strasbourg, 1954).

15. —- Theory of connections, Ann. Mat. Pura Appl. 43 (1957), 119-194.

16. —_, Geometry of bounded domains, Trans. Amer. Math. Soc. 92 (1959), 267-290.

17. M. Kuranishi, On conditions of differentiability of locally compact groups, Nagoya Math. J. 1 (1950), 71-81.

18. D. Montgomery and L. Zippin, Transformation groups, Interscience, New York, 1955.

19. S. Myers and N. Steenrod, The groups of isometries of a Riemannian manifold, Ann. of Math. (2) 40 (1939), 400-416.

20. A. Newlander and L. Nirenberg, Complex analytic coordinates in almost complex manifolds, Ann. of Math. (2) 65 (1957), 391-304.

21. K. Nomizu, On the group of affine transformations of an affinely connected manifold, Proc. Amer. Math. Soc. 4 (1953), 816-823.

22. - Lie groups and differential geometry, The Mathematical Society of Japan, 1956.

23. R. S. Palais. A global formulation of the Lie theory of transformation groups, Mem. Amer. Math. Soc. No. 22 (1957), 123 pp.

24. - On the differentiability of isometries, Proc. Amer. Math. Soc. 8 (1957), 805-807.

25. K. Yano, Some integral formulas and their applications, Michigan Math. J. 5 (1958), 63-73.

UnIVERSiTy OF Alabama Research INSTITUTE, Huntsville, Alabama

UNIVERSITY OF BRITISH COLUMBIA, VANCOUVER, BRITISH COLUMBIA

UNIVERSITY OF CALIFORNIA,

Berkeley, CALifornia 